Article

\title{
Copper Oxide Nanoparticles Cause a Dose-Dependent Toxicity via Inducing Reactive Oxygen Species in Drosophila
}

\author{
Eugene Baeg ${ }^{1}$, Kanidta Sooklert ${ }^{2}$ and Amornpun Sereemaspun ${ }^{2, *}$ \\ 1 Daegu International School, 22 Palgongro, 50-Gil, Donggu, Daegu 701-170, Korea; eugenebaeg@gmail.com \\ 2 Nanomedicine Research Unit, Department of Anatomy, Faculty of Medicine, Chulalongkorn University, \\ Bangkok 10330, Thailand; kanidta.s1986@gmail.com \\ * Correspondence: amornpun.s@chula.ac.th; Tel.: +(66)9-6569-8859
}

Received: 27 September 2018; Accepted: 7 October 2018; Published: 12 October 2018

\begin{abstract}
Copper oxide nanoparticles (CuONPs) have attracted considerable attention, because of their biocide potential and capability for optical imaging, however CuONPs were shown to be highly toxic in various experimental model systems. In this study, mechanism underlying CuONP-induced toxicity was investigated using Drosophila as an in vivo model. Upon oral route of administration, CuONPs accumulated in the body, and caused a dose-dependent decrease in egg-to-adult survivorship and a delay in development. In particular, transmission electron microscopy analysis revealed $\mathrm{CuONPs}$ were detected inside the intestinal epithelial cells and lumen. A drastic increase in apoptosis and reactive oxygen species was also observed in the gut exposed to CuONPs. Importantly, we found that inhibition of the transcription factor Nrf2 further enhances the toxicity caused by CuONPs. These observations suggest that $\mathrm{CuONPs}$ disrupt the gut homeostasis and that oxidative stress serves as one of the primary causes of CuONP-induced toxicity in Drosophila.
\end{abstract}

Keywords: copper oxide nanoparticle; cytotoxicity; reactive oxygen species; Nrf2; Drosophila melanogaster

\section{Introduction}

Nanoparticles (NPs) have unique physicochemical properties caused by their small size, ranging from 1 and $100 \mathrm{~nm}$, and high surface to volume ratio. Hence, applications of manufactured NPs in consumables and biomedical devices are continuously expanding while the increasing use of NPs is associated with potential adverse health concerns [1]. Copper-based NPs have attracted an increased attention for biomedical and industrial applications. For example, smaller CuONPs are fluorescent and have capability for optic imaging, which enables CuONPs to serve as a versatile vehicle for drug delivery and image-guided therapy [2]. CuONPs also show significant antibacterial and biocidal activities, and thus they are being used for the development of many dental and surgical instruments, such as dental composite [3-5]. Furthermore, CuONPs have unique physicochemical properties, such as high electrical and temperature conductivity. Hence, they have been used in magnetic storage media, thermal and electrical devices, sensors, catalysis and semiconductors [6-9]. However, this wide-scale use of CuONPs makes us more prone to their exposure and their potential adverse health effects. In addition, their frequent use provides a negative impact on aquatic ecosystems.

Inhalation is one of the most common routes of metal oxide NP exposure. In the murine model of ovalbumin-induced asthma, CuONPs showed to increase airway hyper-responsiveness, inflammation-related cell number and pro-inflammatory cytokine expression [10], suggesting the pulmonary toxicity of CuONPs. In support of this, recent studies showed that CuONPs facilitate 
pulmonary epithelial cell death and inflammation, eventually leading fibrosis in the C57BL/6 mice model [11]. Furthermore, CuONP exposure was shown to induce fibrotic responses in the mouse respiratory track via up-regulating the expression of transforming growth factor- $\beta 1$ (TGF- $\beta 1$ ) and phosphorylation of Smad3, accompanying with an induction of proinflammatory cytokines, such as interleukin (IL)-1 $\beta$, IL-6 and tumor necrosis factor- $\alpha$ (TNF- $\alpha$ ) [12]. CuONPs have also shown to cause toxic effects in other model organisms. For instance, zebrafish exposed to CuONPs exhibited defects in the liver, neural and cardiac development, dorsoventral patterning and retinal neuro-differentiation $[13,14]$. Acute genotoxic effects and degeneration of dopaminergic neurons by $\mathrm{CuONPs}$ were also reported in the fruit fly Drosophila and C. elegans model, respectively [15-17]. Cytotoxic effects of CuONPs were also reported in various in vitro cellular models. In the human keratinocyte ski HaCaT cells exposed to CuONPs, caspase-3 activity was drastically up-regulated, suggesting that $\mathrm{CuONPs}$ can cause apoptosis [18]. CuONPs are also known to modulate various cellular responses such cell cycle arrest, autophagy and apoptosis in the breast cancer MCF7 cells [19]. Furthermore, CuONPs showed a dose-dependent cyto- and geno-toxicity in the human lung epithelial A549 cells by inducing DNA damage [20], together with a significant reduction in the antioxidant glutathione and induction in caspase-3 activity. Treatment of cultured primary brain astrocytes with CuONPs ( $~ 5 \mathrm{~nm}$ in diameter) was shown to compromise the cell viability in time- and dose-dependent manners [21].

Metal oxide nanoparticles are well known to generate oxidative stress and deregulate normal cellular activities, which subsequently leads to cellular toxicity. Hence, oxidative stress has been considered as one of the primary causes of nanotoxicity and has been reported to use as bio-indicator to evaluate the toxic effects of nanoparticles [22]. In support of this, CuONP exposure was shown to cause a significant accumulation of intracellular reactive oxygen species (ROS) in various cultured cell models and marine model species, such as zebrafish and shrimp [23]. Importantly, quantitative metabolomics and biochemical approaches have revealed that CuONPs induce the regulation of metabolites, such as cysteine-glutathione disulfide and citrulline, which are related to oxidative stress in A549 cells [24], and that the expression and activity of various antioxidant/detoxifying enzymes, such as glutathione peroxidase, superoxide dismutase, glutathione S-transferase, lipid peroxidase and catalase are altered in the mouse hippocampal HT22 cells $[25,26]$. Furthermore, the addition of the antioxidant NAC ( $\mathrm{N}$-acetyl-cysteine) to kidney epithelial cells was shown to mitigate CuONP-induced DNA damage and cell death [27], suggesting the critical role of oxidative stress in CuONP-mediated toxicity. However, direct evidence demonstrating that aberrant ROS are induced by CuONPs in living organism and modulating the ROS levels can indeed modulate toxicity in vivo is still lacking.

The fruit fly Drosophila has a short life cycle of approximately 10-12 days at ambient temperature, distinct development stages; embryonic, larval, pupal and adult stages, high levels of physiological and structural similarity to humans, and a less genetic redundancy compared to mammals. Furthermore, numerous genetic tools and reagents, such as tens of thousands mutant and transgenic lines are easily available for characterizing and dissecting outstanding biological questions. In support of this, Drosophila has received extensive attention for the past decade as a reliable in vivo model organism to understand the fundamental biology of NP-induced toxicity. In this study, we used the fruit fly Drosophila as an in vivo model to examine the potential toxic effects of CuONPs and investigate the underlying mechanism. We found that upon oral route of exposure CuONPs result in a dose-dependent decrease in the egg-to-adult survivorship and delay in development. In particular, ingested CuONPs were detected in the intestinal epithelial cells, which is associated with a drastic induction of cell death and ROS in the gut. Importantly, genetic manipulation of the transcriptional factor Nrf2 (Nuclear factor erythroid 2-related factor 2) activity suggested that intracellular ROS is one of the essential factors that determines the Drosophila viability. 


\section{Materials and Methods}

\subsection{Characterization of $\mathrm{CuONPS}$}

CuONPs (Sigma-Aldrich; CAS no. 1317-38-0, Darmstadt, Germany) of particle size less than $50 \mathrm{~nm}$ (by dynamic light scattering [DLS]) were used in this study. The nanoparticle stock solutions were then diluted to $1 \mathrm{mg} / \mathrm{mL}$ and sterile-filtered before using for assays. The morphology of the CuONPs were studied using transmission electron microscopy (TEM) (JEOL JEM 1010, Tokyo, Japan). TEM samples were prepared by dropping colloidal CuONPs on copper grids pre-coated with Formvar. The Zetasizer Nano was also used to measure the surface charge of the CuONPs.

\subsection{Fly Strains}

Fly stocks were maintained under standard culture conditions. w1118 (a white eyed stock) fly strain was obtained from the Bloomington Drosophila Stock Center and used for toxicity assays, including egg-to-adult survivorship and development. GstD1-green fluorescent protein (GFP) fly line obtained from D. Bohmann was used to monitor the effect of CuONPs on intracellular ROS induction in the gut. To determine whether inhibition of $\mathrm{CncC}$ (the Drosophila homolog of Nrf2), a key transcriptional factor responsible for the expression of antioxidants and detoxifying enzymes, can further increase CuONP-induced intracellular ROS levels and decrease the CuONP-induced toxicity, virgin female CncCVL110/TM3, Sb flies (a gift from D. Bohmann) were crossed with male w1118 flies. Flies were transferred to food containing 0, 0.05 or $0.1 \mathrm{mg} / \mathrm{mL} \mathrm{CuONPs}$. Eclosed F1 adults were collected and counted, and the percentage of viability with respect to control flies with balancer (TM3, Sb allele) was calculated.

\subsection{Drosophila Exposed to CuONPs}

CuONPs at the concentrations of $0,0.05,0.1,0.15$ or $0.25 \mathrm{mg} / \mathrm{mL}$ were added to Drosophila food, which consists of cornmeal flour, dextrose, brewer's yeast, Bacta agar and Nipagin. Drosophila food without CuONPs $(0 \mathrm{mg} / \mathrm{mL})$ was used as a control. Both parental male and female flies were added to vials with or without CuONPs. The flies were then allowed to mate for five days, and then removed. Newly laid eggs (F1 progenies) were maintained in the presence of CuONPs at different concentrations until they emerged (embryonic stage to adult stage).

\subsection{Viability and Development of Drosophila upon CuONPs Treatment}

The effects of $\mathrm{CuONPs}$ on viability were evaluated by counting the number of successfully eclosed F1 flies. The number of days required for the first eclosion was recorded to study the effects of CuONPs on developmental process. Three independent experiments were carried out for statistical analysis.

\subsection{Inductively Coupled Plasma-Mass Spectrometry}

To quantify the amount of CuONPs accumulation in Drosophila, inductively coupled plasma-mass spectrometry (ICP-MS) (Perkin Elmer, USA) was performed. CuONP-fed third instar larvae were homogenized in ultrapure water using a homogenizer to eliminate any source of salt or minerals, which may confound the subsequent analysis and then acid-digested for $24 \mathrm{~h}$ by Aqua Regia. The samples were then diluted with deionized water to appropriate volumes using the Agilent 7500 Series ICP-MS (Perkin Elmer, Norwalk, CT, USA). The final nitric acid was not more than $2 \%$. Three independent experiments were carried out.

\subsection{TEM Study}

To show the accumulation of NPs in the third instar larval intestine upon CuONP ingestion, untreated and CuONP-treated third instar larval midguts were dissected out and collected in phosphate-buffered saline (PBS) for TEM preparation. In brief, the midguts were fixed in $2.5 \%$ 
glutaraldehyde for $1 \mathrm{~h}$, and subsequently osmified with $1 \% \mathrm{OsO} 4$ and bits of $\mathrm{KFeCN}$ at room temperature for $1 \mathrm{~h}$. Samples were then dehydrated and embedded in epoxy resin (polymerization at $60{ }^{\circ} \mathrm{C}$ for overnight) (Ted Pella Inc., Redding, CA, USA), followed by slicing of ultrathin sections. The sections were subsequently stained with lead citrate (BDH, Bristol, UK). Digital micrographs were obtained using a Gatan 792 Bioscan $1 \mathrm{k} \times 1 \mathrm{k}$ Wide Angle Multiscan charge-coupled device camera attached to the JEOL 1010 TEM (Tokyo, Japan).

\subsection{Cellular ROS Detection Assay}

In Drosophila, CncC regulates the expression of gstD1 (glutathione $\mathrm{S}$ transferase D1), which encodes a detoxification enzyme. To monitor the effects of CuONPs on ROS induction in the gut, transgenic flies carrying an oxidative stress reporter gene gstD1-GFP were used to assess whether CuONP exposure can induce the oxidative stress reporter activity. The third instar larval midguts were dissected in dissecting solution pH $7.2\left(130 \mathrm{mM} \mathrm{NaCl} ; 1.9 \mathrm{mM} \mathrm{CaCl}_{2} ; 4.7 \mathrm{mM} \mathrm{KCl}\right.$; $10 \mathrm{mM}$ HEPES) and observed under the confocal microscope (Olympus Fluoview FV1000 cLSM, Tokyo, Japan) to monitor GFP expression. To further confirm the induction of ROS upon CuONPs, the dissected guts were also stained with $10 \mu \mathrm{M}$ dihydroethidium (DHE) after fixation for $5 \mathrm{~min}$ and then washed in PBST three times. The guts were then mounted on Vectashield mounting medium containing DAPI on a slide glass and viewed under the confocal microscope.

\subsection{Terminal Deoxynucleotidyl Transferase dUTP Nick End Labelling (TUNEL)-Assay of the Drosophila Gut}

The guts treated with or without CuONPs were subjected to Terminal deoxynucleotidyl transferase dUTP nick end labelling (TUNEL) assay (catalog number 11684795910; Roche Applied Science, Mannheim, Germany). Briefly, samples were fixed in $4 \%$ paraformaldehyde, permeabilized with $0.1 \%$ Triton $\mathrm{X}-100$ in $0.1 \%$ sodium citrate solution followed by labeling with TUNEL reaction mixture.

\subsection{Statistical Analysis}

Statistical analyses were performed using the GraphPad Prism software (version 6.0). Values from all experiments were expressed in mean \pm standard error. The data was analyzed by unpaired $t$-test or one-way ANOVA with post-hoc test (Tukey's Multiple Comparison Test). $p<0.05$ was statistically significant.

\section{Results and Discussion}

\subsection{Characterization of $\mathrm{CuONPs}$}

The size and morphology of CuONPs used in this study were examined using transmission electron microscopy (TEM). TEM micrographs showed that CuONPs are spherical in shape and uniform homogenously dispersed in suspension, with the defined size of approximately $40 \mathrm{~nm}$ (Figure 1a). Consistently, the size distribution of $\mathrm{CuO}$ particles, which was determined by ImageJ software showed that the average diameter of CuONPs is $\sim 40 \mathrm{~nm}$, as observed under TEM (Figure 1b). Lastly, the zeta potential of the CuONPs was found to be $-28.1 \mathrm{mV}$, suggesting that they are relatively stable (Figure 1c). As shown in Figure 1a, agglomeration of CuONPs was observed, and thus freshly sonicated $\mathrm{CuONP}$ dispersions were used immediately for assays. 

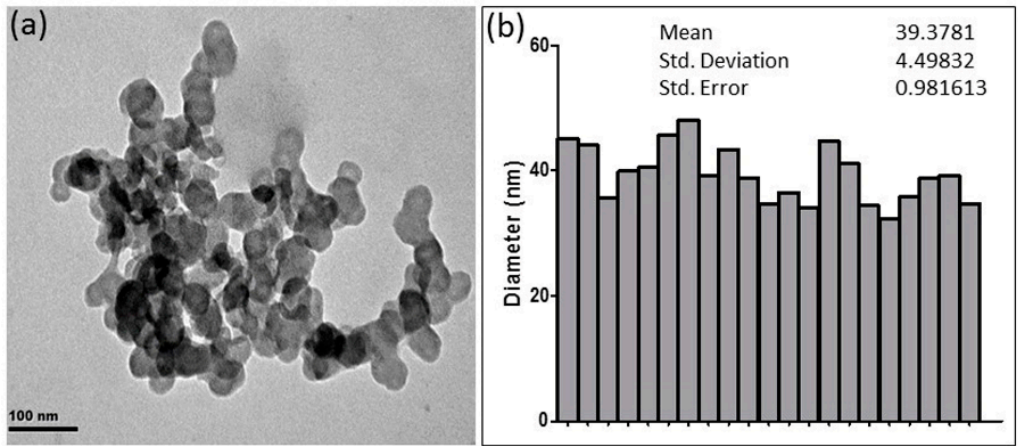

(c)

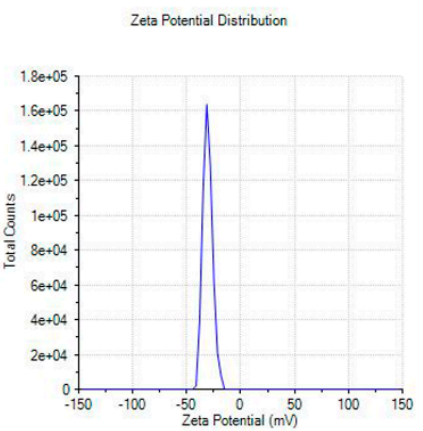

Figure 1. Characterization of Copper oxide nanoparticles (CuONPs). (a) Transmission electron microscopy (TEM) micrographs show spherical CuONPs with the size of approximately $40 \mathrm{~nm}$; (b) The size distribution of CuONPs was determined by ImageJ software; (c) The zeta potential of CuONPs is $-28.1 \mathrm{mV}$.

\subsection{CuONPs Cause Toxic Effects in the Fruit Fly Drosophila}

To examine the potential toxic effects of CuONPs with the size of $\sim 40 \mathrm{~nm}$ in Drosophila, we first monitored the uptake and accumulation of CuONPs in vivo after oral route of administration. F1 (first filial or generation) progenies derived from the control parental w1118 flies were maintained in food in the presence of CuONPs at various concentrations, ranging from 0.05 to $0.1 \mathrm{mg} / \mathrm{mL}$. We then performed the inductively coupled plasma mass spectrometry (ICP-MS) analysis using CuONP-fed third instar larvae to show that CuONPs indeed accumulate in the body. Five late third instar larvae were collected from each experimental group, homogenized, and then acid-digested. The concentration of CuONPs per parts per billion (ppb), which indicates the number of units of mass of a contaminant per 1000 million units of total mass was determined. As shown in Figure 2a, upon exposure to CuONPs at the concentration of $0.05 \mathrm{mg} / \mathrm{mL}$, a drastic accumulation of CuONPs was observed compared to control. A significant CuONP accumulation at the concentration of $0.1 \mathrm{mg} / \mathrm{mL}$ was also observed, but the significance was reduced compared to that observed at $0.05 \mathrm{mg} / \mathrm{mL}$. Interestingly, we noticed that late third instar larvae exposed to CuONPs at higher doses, such as $0.1 \mathrm{mg} / \mathrm{mL}$ were much smaller than those at $0.05 \mathrm{mg} / \mathrm{mL}$. This suggests that higher doses of CuONPs severely disrupted the metabolism of the larvae, raising the possibility that larvae exposed to the higher doses of CuONPs stopped ingesting food containing CuONPs at early larval stages whereas larvae exposed to lower concentrations continued to ingest food and accumulate CuONPs throughout the entire larval stages. In support of this, we also observed that ingestion of CuONPs causes a dose-dependent decrease in the number of pupa (Figure $2 b$ ). Hence, we monitored the egg-to-adult survivorship upon CuONP exposure. The number of F1 flies successfully eclosed to adult was found to be dose-dependently decreased (Figure 2c). In particular, a significant reduction of the survivorship was observed upon higher doses of CuONPs (such as $0.15 \mathrm{mg} / \mathrm{mL}$ or $0.25 \mathrm{mg} / \mathrm{mL}$ ) as compared to untreated control flies. Furthermore, we found that treatment of flies with CuONPs also results in a significant delay in the developmental process of Drosophila. As shown in Figure 2d, F1 flies exposed to CuONPs at higher concentrations showed a drastically delayed eclosion compared to those exposed to control or $0.05 \mathrm{mg} / \mathrm{mL}$ CuONPs. 
(a)

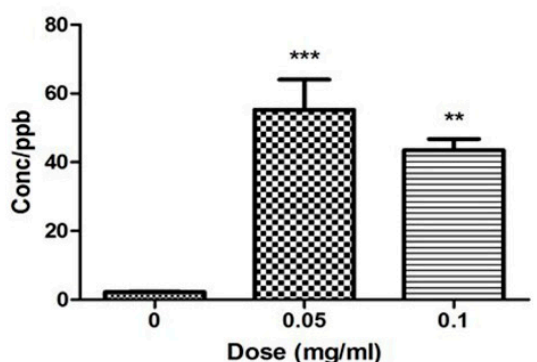

(c)

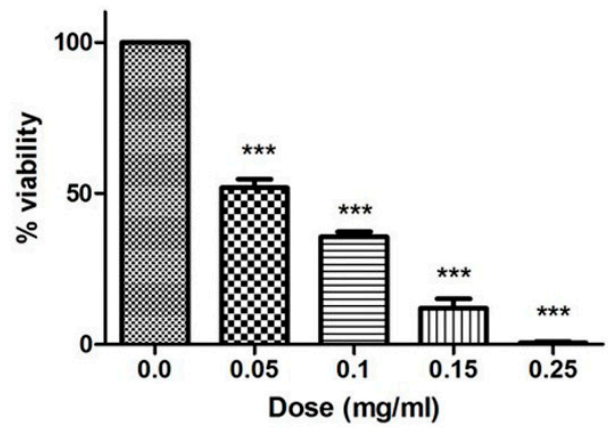

(b)

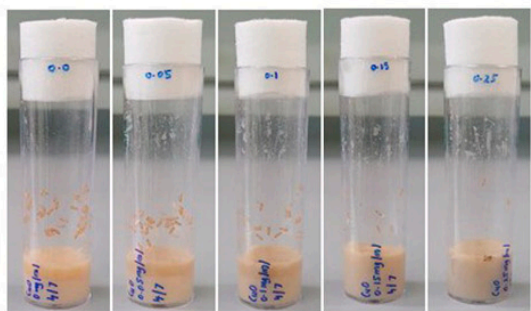

(d)

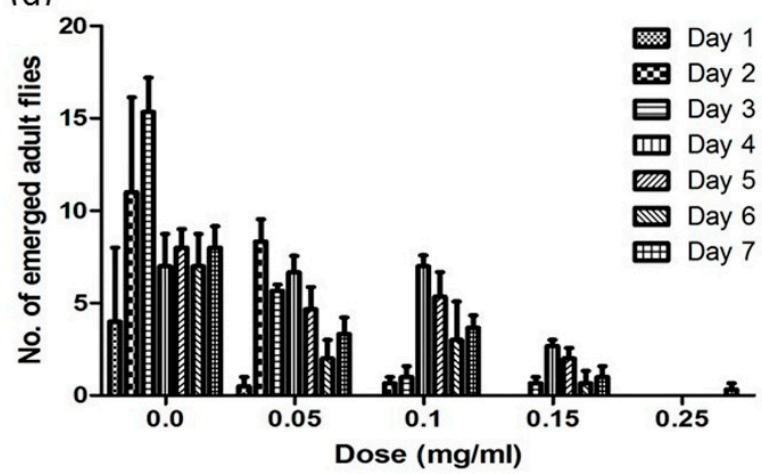

Figure 2. CuONP ingestion induces toxic effects in Drosophila. (a) CuONP-fed F1 progenies show an uptake and accumulation of CuONPs in the body. $(\mathbf{b}, \mathbf{c})$ A dose-dependent decline in the number of pupa and in egg-to-adult survivorship is observed upon CuONPs. (d) CuONP exposure causes a dose-dependent delay in development. Error bar $=\mathrm{SEM},{ }^{*} p$-value $<0.05$; ${ }^{*} p$-value $<0.01$; *** $p$-value $<0.001$

These findings are in accord with previous reports that treatment of CuONPs can cause cytotoxicity in Drosophila, as well as in other model systems, such as cultured cell lines, C. elegans and marine model species $[16,18,21,28]$. Notably, contribution of NPs and their released $\mathrm{Cu}^{2+}$ leached from the NPs to the overall toxicity of $\mathrm{CuONPs}$ is still not clear. For instance, measurement of $\mathrm{Cu}^{2+}$ released from CuONPs in cell culture medium suggested that $\mathrm{Cu}^{2+}$ cations contribute only to a small extent in CuONP-induced toxicity on HepG2 cells [29]. Another study reported that CuONPs can generate significantly more ROS and DNA damage than dissolved $\mathrm{Cu}^{2+}$ ions, suggesting that the surface of NPs plays key roles in inducing toxicity [30]. By contrast, CuONP-associated toxicity was reported to be predominantly mediated by dissolved $\mathrm{Cu}^{2+}$ ions in A549 and the lung epithelial BEAS-2B cells [31]. Furthermore, more severe genotoxic effects were detected from dissolved $\mathrm{Cu}^{2+}$ ions than those from $\mathrm{CuONPs}$ in Drosophila, suggesting that $\mathrm{Cu}^{2+}$ ions play more important role than physicochemical properties of NPs in inducing toxic effects $[15,16]$. Hence, more comprehensive analyses with developed techniques are required to differentiate toxicities caused by CuONPs or their dissolved $\mathrm{Cu}^{2+}$ ions, which will be of great help to generate hypotheses on how to minimize or abolish the toxic effects of CuONPs and establish a risk assessment of $\mathrm{CuONPs}$ via modulating their physicochemical properties, selecting proper route of administration and controlling the release $\mathrm{Cu}^{2+}$ ions.

\subsection{Accumulation of $\mathrm{CuONPs}$ in the Gut}

NPs are also being used in food industry to improve color, texture and flavor of food [32]. In addition, nanotechnology is now widely accepted and used to improve the delivery of orally administered drugs, implying that the gastrointestinal track is one of the initial organ systems affected by dietary NPs. Indeed, it was suggested that NPs enter and accumulate in the intestine, and subsequently translocate across the intestinal barrier via a few possible mechanisms, including endocytosis, persorption, and putative para-cellular uptake of NPs [33]. Furthermore, a previous study using Drosophila suggested that CuONPs can travel inside the midgut epithelial cells and cross over the intestinal barrier to interact with circulating hemolymph, which is equivalent to the blood in 
mammals [16]. We thus examined the distribution and localization of NPs in the midgut of third instar larvae fed with CuONPs by TEM after ultrathin sectioning. In the control intestinal epithelial cells, CuONPs with the size of 40-50 m were not detected (Figure 3a). However, in the CuONP-exposed epithelial cells CuONPs were found in the cytosol, as well as inside the vesicle (Figure $3 b$ ).
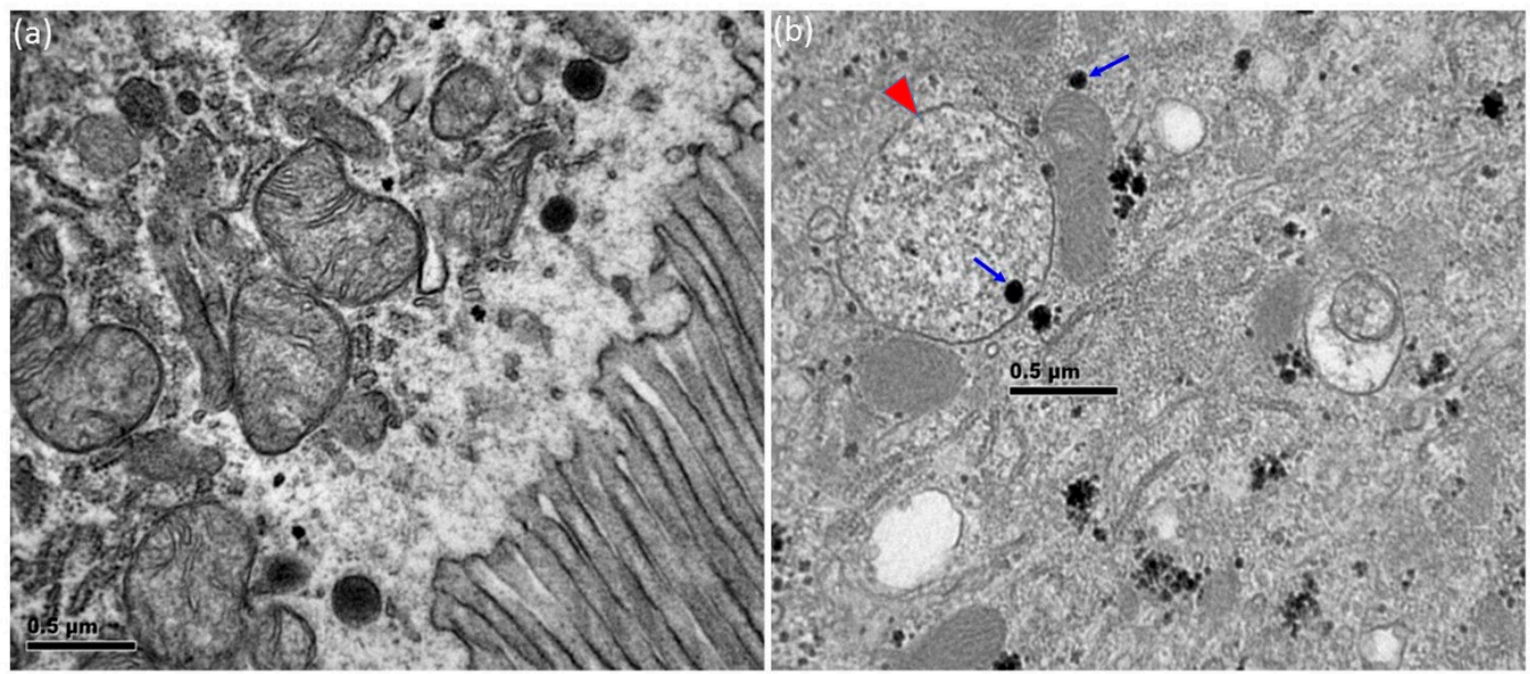

Figure 3. Accumulation of CuONPs in the intestinal epithelial cells. (a) CuONPs with the size of 40-50 $\mathrm{nm}$ are not detected in the control intestinal epithelial cells; (b) CuONPs (arrows) are detected inside the cytoplasm of CuONP-exposed epithelia cells. Note that some of CuONPs are observed inside the vesicle (arrowhead).

\subsection{CuONPs Induce Cell Death and Oxidative Stress in the Gut}

Toxicological effects of particles, such as AgNPs and ZnONPs in the human intestinal cell line Caco-2 were reported. NP exposure was found to induce mitochondrial and DNA damage, cell membrane leakage and inflammation, resulting in cell-cycle arrest and subsequently cell death [34-36]. Since we observed a significant decrease in viability and delay in development upon CuONP exposure (Figure 2c,d), we examined whether exposure to CuONPs can cause cell death of the intestinal epithelial cells by performing TUNEL assay. In the untreated gut, TUNEL-positive epithelial cells were barely detected (Figure $4 \mathrm{a}, \mathrm{a}^{\prime}$ ). However, exposure to $\mathrm{CuONPs}$ resulted in a dose-dependent increase in the number of TUNEL-positive cells in the midgut, suggesting that the gut epithelial cells underwent apoptosis upon CuONPs (Figure $4 b, b^{\prime}, c, c^{\prime}$ ). Interestingly, it was shown that NPs, such as AgNPs, $\mathrm{TiO}_{2} \mathrm{NPs}$ and $\mathrm{SiO}_{2} \mathrm{NPs}$ are known to disrupt the gut microbiota in the mouse model [37-39]. Furthermore, dietary exposure to AgNPs resulted in an increase in Gram-positive genera, in particular Lactobacillus, in the midgut of Drosophila, and toxicity of NPs within the gut was considered to be responsible for developmental delay and decreased survivorship [40], suggesting that CuONPs may not only induce apoptosis of epithelial cells, but also disrupt microbiota in the gut, leading to poor survivorship and delayed development. 


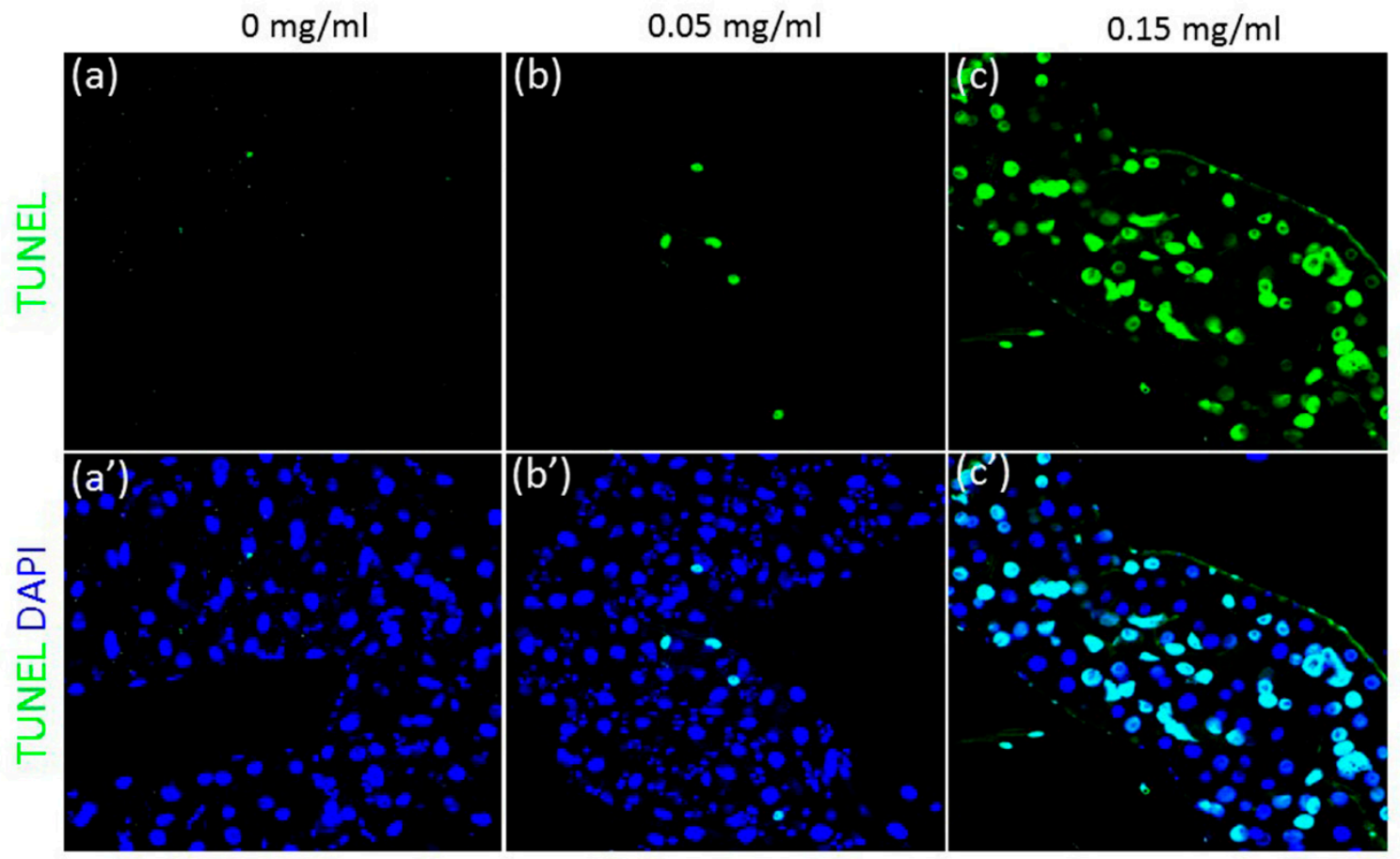

Figure 4. CuONP exposure causes apoptosis of the gut epithelia cells. Flies were exposed to CuONPs at the concentration of $\left(\mathbf{a}, \mathbf{a}^{\prime}\right) 0 \mathrm{mg} / \mathrm{mL},\left(\mathbf{b}, \mathbf{b}^{\prime}\right) 0.05 \mathrm{mg} / \mathrm{mL}$ and $\left(\mathbf{c}, \mathbf{c}^{\prime}\right) 0.15 \mathrm{mg} / \mathrm{mL}$. The third instar larval guts show a dose-dependent increase in the number of terminal deoxynucleotidyl transferase dUTP nick end labelling (TUNEL)-positive epithelial cells (green).

Oxidative stress is considered to be one of the primary causes of cytotoxicity, genotoxicity, inflammation, cell death and altered nutrient absorption induced by NP exposure in the gut [41-43]. We have also previously shown that AgNP ingestion causes excessive intracellular ROS induction in the Drosophila testis, leading to oxidative stress responsible for defects in germline stem cell homeostasis and a significant decrease in male fecundity [44]. All these observations suggest that NP-induced oxidative stress may cause a defect in the function of macromolecules, such as protein and lipid, leading to its detrimental toxic effects on various organ systems. Hence, we examined the effects of CuONPs on ROS induction in the gut of third instar larvae. We found that CuONPs greatly increase ROS levels. Dihydroethidium (DHE) probe was used to monitor ROS, particularly superoxide (O2-) levels, as it readily reacts with superoxide anions to form 2-hydroxyethidium, generating red fluorescence [45]. In the control gut of larvae exposure to control CuONPs, basal levels of ROS were detected in the gut epithelial cells (Figure 5a, $\mathrm{a}^{\prime}$ ). However, a dose-dependent increase in DHE expression was detected upon CuONP exposure (Figure $\left.5 b, b^{\prime}, c, c^{\prime}\right)$. To further confirm the effects of $\mathrm{CuONPs}$ on ROS induction, we next used transgenic flies carrying the independent oxidative stress reporter gene GstD1-GFP and assessed whether CuONP exposure can enhance the reporter activity in the gut epithelial cells. Upon oxidative stress, the transcriptional factor Nrf2, possibly together with small-Maf, binds to antioxidant response element (ARE) within the promoter region of the antioxidant gene GstD1, leading to an increase in the expression of GstD1 [46]. Hence, high levels of GFP expression are expected upon oxidative stress. In accordance with the findings obtained from DHE staining, only basal levels of GFP were detected in the control gut epithelial cells (Figure 5d,d'). However, we observed a dose-dependent increase in GFP expression in the epithelial cells upon $\mathrm{CuONP}$ exposure (Figure $\left.5 \mathrm{e}, \mathrm{e}^{\prime}, \mathrm{f}, \mathrm{f}^{\prime}\right)$. These observations strongly suggest that ingested CuONP induced oxidative stress, which is closely associated with apoptosis of the gut epithelial cells, and decreased viability and delayed development of the organism. 


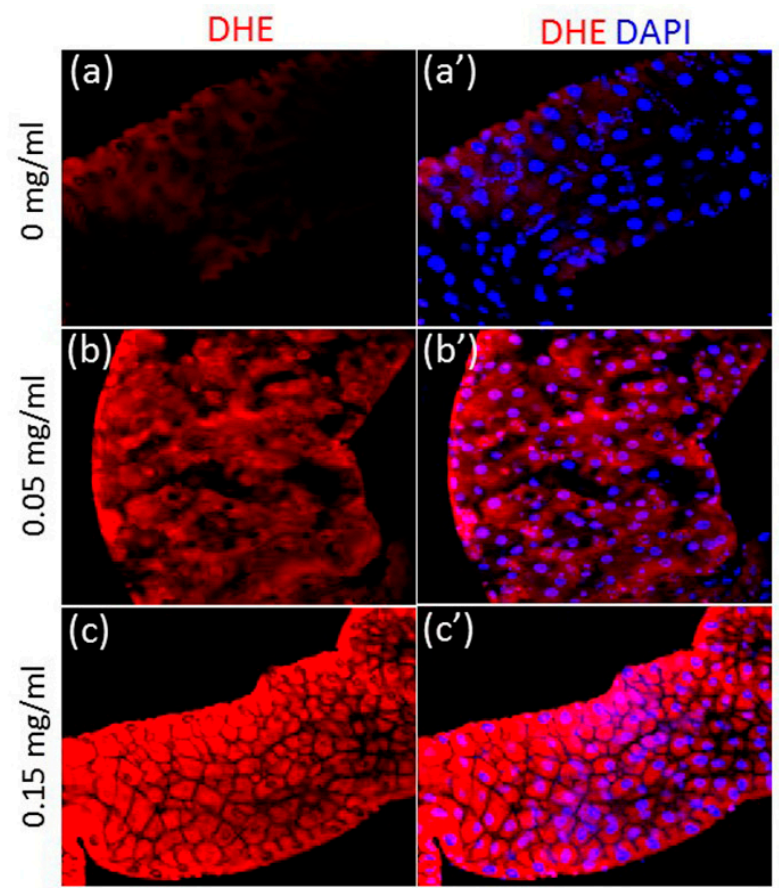

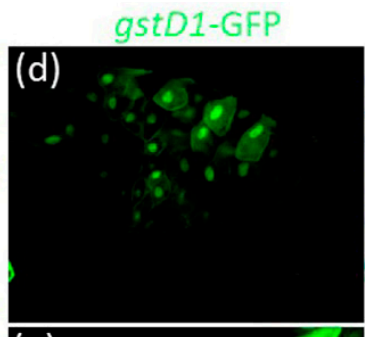

gstD1-GFP DAPI
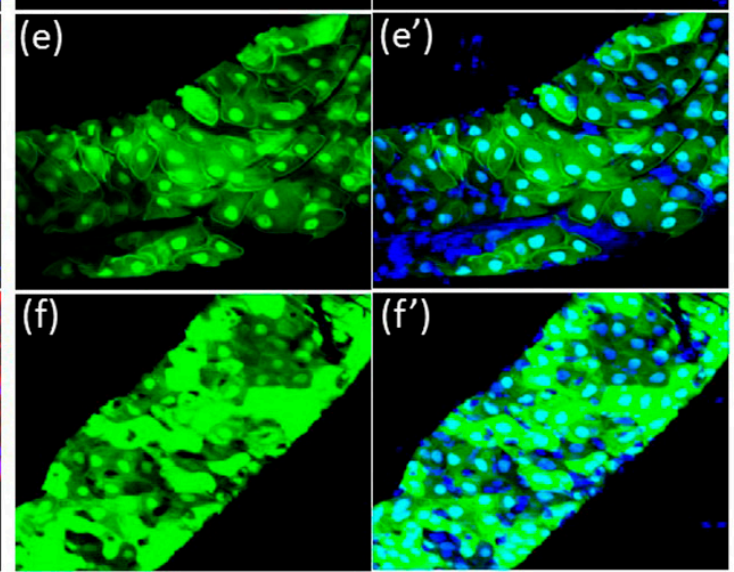

Figure 5. CuONPs induce excessive reactive oxygen species (ROS) production in the gut. Flies were exposed to CuONPs at the concentration of $\left(\mathbf{a}, \mathbf{a}^{\prime}, \mathbf{d}, \mathbf{d}^{\prime}\right) 0 \mathrm{mg} / \mathrm{mL},\left(\mathbf{b}, \mathbf{b}^{\prime}, \mathbf{e}, \mathbf{e}^{\prime}\right) 0.05 \mathrm{mg} / \mathrm{mL}$ and $\left(\mathbf{c}, \mathbf{c}^{\prime}, \mathbf{f}, \mathbf{f}^{\prime}\right)$ $0.15 \mathrm{mg} / \mathrm{mL}$. (a, $\left.\mathbf{a}^{\prime}\right)$ Dihydroethidium (DHE) staining that detects superoxide levels shows the basal levels of ROS in the control gut epithelial cells. $\left(\mathbf{b}, \mathbf{b}^{\prime}, \mathbf{c}, \mathbf{c}^{\prime}\right)$ ROS levels become gradually increased upon $\mathrm{CuONPs}$ in a dose-dependent manner. Transgenic flies carrying the oxidative stress reporter gene GstD1-GFP were used to monitor the effects of CuONPs on intracellular ROS induction. (d, d') The control gut shows weak GFP expression. $\left(\mathbf{e}, \mathbf{e}^{\prime}, \mathbf{f}, \mathbf{f}^{\prime}\right)$ GFP expression is dose-dependently increased upon CuONPs.

\subsection{Inhibition of Nrf2 Further Decreases the Poor Survivorship Caused by CuONPs}

To demonstrate that the decreased viability in CuONP-treated flies was at least in partly associated with excessive ROS induction, we examined the effects of loss-of-function of Nrf2 on the decreased viability. Keap1/Nrf2 complex plays as an important cellular sensor for oxidative stress [47,48]. Under the normal condition, the transcriptional factor Nrf2 is involved in the cellular response to oxidative and electrophilic stress [49], and is negatively regulated by Keap1 via ubiquitination in the cytoplasm [50]. However, upon oxidative stress by oxidants or electrophilic insults, Nrf2 becomes activated by protein stabilization, translocates into the nucleus to engage in the transcriptional activation of genes encoding a large pool of antioxidant and phase II detoxifying enzymes, including superoxide dismutase (SOD), catalase and glutathione peroxidase (GTPx), to scavenge excessive intracellular ROS (Figure 6a). Thus, we reasoned that CuONP-induced fly lethality would be even more severe when the activity of Nrf2 is down-regulated. Nrf2 activity was genetically manipulated in both control and CuONP-fed F1 progenies by introducing one copy of CncC (the Drosophila homolog of Nrf2) mutant alleles (CncCK6), and the decreased viability caused by CuONPs was monitored. As expected, CuONP-induced poor viability was further decreased in $\mathrm{CncC}+/$ - heterozygous flies compared to control flies (Figure 6b). This provides compelling evidence that oxidative stress is one of the direct causes of CuONP-induced toxicity and that antioxidants or detoxifying enzymes play essential roles in salvaging ROS-associated toxicity upon CuONP exposure in Drosophila. 
(a)

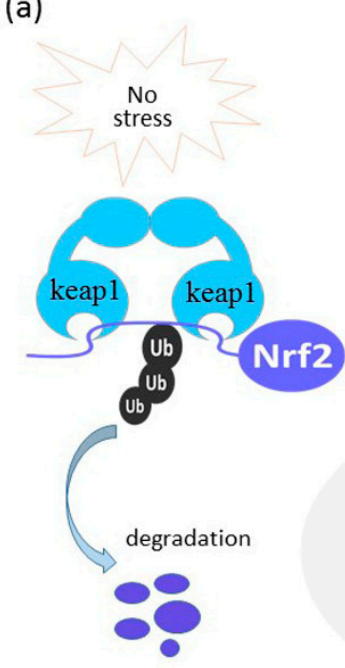

(b)

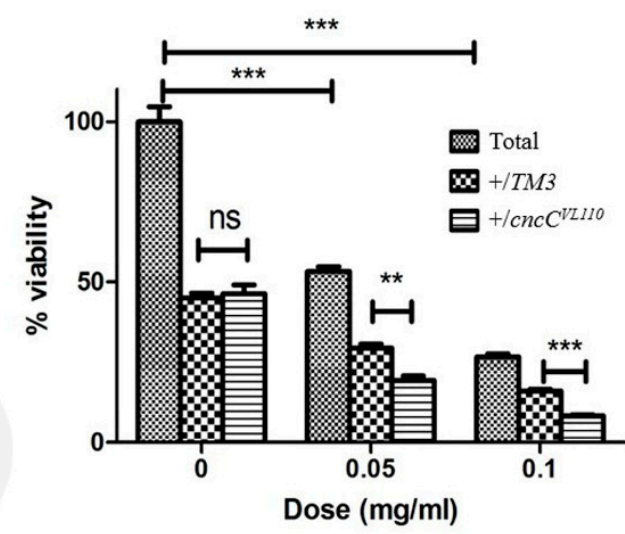

Figure 6. ROS levels modulate the toxic effects caused by CuONPs. (a) Schematic diagram suggesting the role of Nrf2 in the regulation of antioxidant gene expression; (b) Introducing one mutant allele of CncC (the Drosophila homolog of Nrf2) further decreased the poor survivorship caused by CuONP exposure. Error bar $=\mathrm{SEM},{ }^{* *} p$-value $<0.01 ;{ }^{* * *} p$-value $<0.001$.

\section{Conclusions}

The oral route of CuONP administration has resulted in a significant toxicity in Drosophila at the organism level. Specifically, CuONP exposure caused a decline in egg-to-adult survivorship and a delay in development of CuONP-fed offsprings. Furthermore, CuONPs induced a drastic ROS production in the Drosophila gut, which was possibly related to an increase in apoptosis of the gut epithelial cells. Importantly, more significant decreased viability was observed in $\mathrm{CncC}+\mathrm{-}$ flies compared to that of in control flies (carrying a balancer allele) upon CuONPs, indicating the essential role of ROS levels in CuONP-mediated toxicity in vivo. Nonetheless, limited understanding of the mechanisms of $\mathrm{CuONP}$-associated toxicity would need further elucidation to have meaningful assessment of nanosafety. Furthermore, comprehensive understanding of underlying mechanisms is of great importance to assess the environmental risk of CuONPs and to expand their use safely.

Author Contributions: E.B. conducted experiments and performed data analysis. K.S. collected and analyzed data. A.S. conceived the study, provided inputs to experimental protocols and wrote the manuscript. All authors read and approved the final manuscript before submission.

Funding: This research was funded by the Chulalongkorn University; Government Budget and the Grant to support a research group in the Ratchadaphiseksomphot Endowment Fund, Chulalongkorn University, and partially supported by the Ratchadaphiseksomphot Endowment Fund of Chulalongkorn University (RES560530230-AM).

Acknowledgments: The authors would like to thank Song-Lin Bay for her assistance in preparing the artwork for the figure used in this study. K.S. would like to acknowledge her postdoctoral fellowship supported by the Ratchadapiseksompote Endowment Fund, Chulalongkorn University.

Conflicts of Interest: The authors declare no conflict of interest.

\section{References}

1. Pathakoti, K.; Manubolu, M.; Hwang, H.-M. Chapter 48-Nanotechnology applications for environmental industry. In Handbook of Nanomaterials for Industrial Applications; Hussain, C.M., Ed.; Elsevier: Cambridge, MA, USA, 2018; pp. 894-907.

2. Zhou, M.; Tian, M.; Li, C. Copper-Based Nanomaterials for Cancer Imaging and Therapy. Bioconjugate Chem. 2016, 27, 1188-1199. [CrossRef] [PubMed] 
3. Hannig, M.; Kriener, L.; Hoth-Hannig, W.; Becker-Willinger, C.; Schmidt, H. Influence of nanocomposite surface coating on biofilm formation in situ. J. Nanosci. Nanotechnol. 2007, 7, 4642-4648. [PubMed]

4. Monteiro, D.R.; Gorup, L.F.; Takamiya, A.S.; Ruvollo-Filho, A.C.; de Camargo, E.R.; Barbosa, D.B. The growing importance of materials that prevent microbial adhesion: Antimicrobial effect of medical devices containing silver. Int. J. Antimicrob. Agents 2009, 34, 103-110. [CrossRef] [PubMed]

5. Nations, S.; Long, M.; Wages, M.; Maul, J.D.; Theodorakis, C.W.; Cobb, G.P. Subchronic and chronic developmental effects of copper oxide $(\mathrm{CuO})$ nanoparticles on Xenopus laevis. Chemosphere 2015, 135, 166-174. [CrossRef] [PubMed]

6. Zhang, Q.; Zhang, K.; Xu, D.; Yang, G.; Huang, H.; Nie, F.; Liu, C.; Yang, S. CuO nanostructures: Synthesis, characterization, growth mechanisms, fundamental properties, and applications. Prog. Mater. Sci. 2014, 60, 208-337. [CrossRef]

7. Devi, A.B.; Moirangthem, D.S.; Talukdar, N.C.; Devi, M.D.; Singh, N.R.; Luwang, M.N. Novel synthesis and characterization of $\mathrm{CuO}$ nanomaterials: Biological applications. Chin. Chem. Lett. 2014, 25, 1615-1619. [CrossRef]

8. Dagher, S.; Haik, Y.; Ayesh, A.I.; Tit, N. Synthesis and optical properties of colloidal CuO nanoparticles. J. Lumin. 2014, 151, 149-154. [CrossRef]

9. Perlman, O.; Weitz, I.S.; Azhari, H. Copper oxide nanoparticles as contrast agents for MRI and ultrasound dual-modality imaging. Phys. Med. biol. 2015, 60, 5767-5783. [CrossRef] [PubMed]

10. Park, J.W.; Lee, I.C.; Shin, N.R.; Jeon, C.M.; Kwon, O.K.; Ko, J.W.; Kim, J.C.; Oh, S.R.; Shin, I.S.; Ahn, K.S. Copper oxide nanoparticles aggravate airway inflammation and mucus production in asthmatic mice via MAPK signaling. Nanotoxicology 2016, 10, 445-452. [CrossRef] [PubMed]

11. Lai, X.; Zhao, H.; Zhang, Y.; Guo, K.; Xu, Y.; Chen, S.; Zhang, J. Intranasal Delivery of Copper Oxide Nanoparticles Induces Pulmonary Toxicity and Fibrosis in C57BL/6 mice. Sci. Rep. 2018, 8, 4499. [CrossRef] [PubMed]

12. Ko, J.W.; Shin, N.R.; Park, J.W.; Park, S.H.; Lee, I.C.; Kim, J.S.; Kim, J.C.; Ahn, K.S.; Shin, I.S. Copper oxide nanoparticles induce collagen deposition via TGF-beta1/Smad3 signaling in human airway epithelial cells. Nanotoxicology 2018, 12, 239-250. [CrossRef] [PubMed]

13. Xu, J.; Zhang, Q.; Li, X.; Zhan, S.; Wang, L.; Chen, D. The effects of copper oxide nanoparticles on dorsoventral patterning, convergent extension, and neural and cardiac development of zebrafish. Aquat. Toxicol. 2017, 188, 130-137. [CrossRef] [PubMed]

14. Sun, Y.; Zhang, G.; He, Z.; Wang, Y.; Cui, J.; Li, Y. Effects of copper oxide nanoparticles on developing zebrafish embryos and larvae. Int. J. Nanomed. 2016, 11, 905-918. [CrossRef]

15. Carmona, E.R.; Inostroza-Blancheteau, C.; Obando, V.; Rubio, L.; Marcos, R. Genotoxicity of copper oxide nanoparticles in Drosophila melanogaster. Mutat. Res.-Genet. Toxicol. Eviron. Mutagen. 2015, 791, 1-11. [CrossRef] [PubMed]

16. Alaraby, M.; Hernandez, A.; Marcos, R. New insights in the acute toxic/genotoxic effects of $\mathrm{CuO}$ nanoparticles in the in vivo Drosophila model. Nanotoxicology 2016, 10, 749-760. [CrossRef] [PubMed]

17. Mashock, M.J.; Zanon, T.; Kappell, A.D.; Petrella, L.N.; Andersen, E.C.; Hristova, K.R. Copper Oxide Nanoparticles Impact Several Toxicological Endpoints and Cause Neurodegeneration in Caenorhabditis elegans. PLoS ONE 2016, 11, e0167613. [CrossRef] [PubMed]

18. Alarifi, S.; Ali, D.; Verma, A.; Alakhtani, S.; Ali, B.A. Cytotoxicity and genotoxicity of copper oxide nanoparticles in human skin keratinocytes cells. Int. J. Toxicol. 2013, 32, 296-307. [CrossRef] [PubMed]

19. Laha, D.; Pramanik, A.; Maity, J.; Mukherjee, A.; Pramanik, P.; Laskar, A.; Karmakar, P. Interplay between autophagy and apoptosis mediated by copper oxide nanoparticles in human breast cancer cells MCF7. Biochim. Biophys. Acta 2014, 1840, 1-9. [CrossRef] [PubMed]

20. Akhtar, M.J.; Kumar, S.; Alhadlaq, H.A.; Alrokayan, S.A.; Abu-Salah, K.M.; Ahamed, M. Dose-dependent genotoxicity of copper oxide nanoparticles stimulated by reactive oxygen species in human lung epithelial cells. Toxicol. Ind. Health 2016, 32, 809-821. [CrossRef] [PubMed]

21. Bulcke, F.; Thiel, K.; Dringen, R. Uptake and toxicity of copper oxide nanoparticles in cultured primary brain astrocytes. Nanotoxicology 2014, 8, 775-785. [CrossRef] [PubMed]

22. Libralato, G.; Galdiero, E.; Falanga, A.; Carotenuto, R.; de Alteriis, E.; Guida, M. Toxicity Effects of Functionalized Quantum Dots, Gold and Polystyrene Nanoparticles on Target Aquatic Biological Models: A Review. Molecules 2017, 22, 1439. [CrossRef] [PubMed] 
23. Ivask, A.; Juganson, K.; Bondarenko, O.; Mortimer, M.; Aruoja, V.; Kasemets, K.; Blinova, I.; Heinlaan, M.; Slaveykova, V.; Kahru, A. Mechanisms of toxic action of $\mathrm{Ag}, \mathrm{ZnO}$ and $\mathrm{CuO}$ nanoparticles to selected ecotoxicological test organisms and mammalian cells in vitro: A comparative review. Nanotoxicology 2014, 8 , 57-71. [CrossRef] [PubMed]

24. Boyles, M.S.; Ranninger, C.; Reischl, R.; Rurik, M.; Tessadri, R.; Kohlbacher, O.; Duschl, A.; Huber, C.G. Copper oxide nanoparticle toxicity profiling using untargeted metabolomics. Part. Fibre Toxicol. 2016, 13, 49. [CrossRef] [PubMed]

25. Niska, K.; Santos-Martinez, M.J.; Radomski, M.W.; Inkielewicz-Stepniak, I. CuO nanoparticles induce apoptosis by impairing the antioxidant defense and detoxification systems in the mouse hippocampal HT22 cell line: Protective effect of crocetin. Toxicol. In Vitro 2015, 29, 663-671. [CrossRef] [PubMed]

26. Thit, A.; Selck, H.; Bjerregaard, H.F. Toxic mechanisms of copper oxide nanoparticles in epithelial kidney cells. Toxicol. In Vitro 2015, 29, 1053-1059. [CrossRef] [PubMed]

27. Srikanth, K.; Pereira, E.; Duarte, A.C.; Rao, J.V. Evaluation of cytotoxicity, morphological alterations and oxidative stress in Chinook salmon cells exposed to copper oxide nanoparticles. Protoplasma 2016, 253, 873-884. [CrossRef] [PubMed]

28. Rotini, A.; Gallo, A.; Parlapiano, I.; Berducci, M.T.; Boni, R.; Tosti, E.; Prato, E.; Maggi, C.; Cicero, A.M.; Migliore, L.; et al. Insights into the $\mathrm{CuO}$ nanoparticle ecotoxicity with suitable marine model species. Ecotoxicol. Environ. Saf. 2018, 147, 852-860. [CrossRef] [PubMed]

29. Piret, J.P.; Jacques, D.; Audinot, J.N.; Mejia, J.; Boilan, E.; Noel, F.; Fransolet, M.; Demazy, C.; Lucas, S.; Saout, C.; et al. Copper(II) oxide nanoparticles penetrate into HepG2 cells, exert cytotoxicity via oxidative stress and induce pro-inflammatory response. Nanoscale 2012, 4, 7168-7184. [CrossRef] [PubMed]

30. Cronholm, P.; Karlsson, H.L.; Hedberg, J.; Lowe, T.A.; Winnberg, L.; Elihn, K.; Wallinder, I.O.; Moller, L. Intracellular uptake and toxicity of $\mathrm{Ag}$ and $\mathrm{CuO}$ nanoparticles: A comparison between nanoparticles and their corresponding metal ions. Small 2013, 9, 970-982. [CrossRef] [PubMed]

31. Angele-Martinez, C.; Nguyen, K.V.; Ameer, F.S.; Anker, J.N.; Brumaghim, J.L. Reactive oxygen species generation by copper(II) oxide nanoparticles determined by DNA damage assays and EPR spectroscopy. Nanotoxicology 2017, 11, 278-288. [CrossRef] [PubMed]

32. Pathakoti, K.; Manubolu, M.; Hwang, H.-M. Nanostructures: Current uses and future applications in food science. J. Food Drug Anal. 2017, 25, 245-253. [CrossRef] [PubMed]

33. Powell, J.J.; Faria, N.; Thomas-McKay, E.; Pele, L.C. Origin and fate of dietary nanoparticles and microparticles in the gastrointestinal tract. J. Autoimmun. 2010, 34, J226-J233. [CrossRef] [PubMed]

34. Bohmert, L.; Niemann, B.; Thunemann, A.F.; Lampen, A. Cytotoxicity of peptide-coated silver nanoparticles on the human intestinal cell line Caco-2. Arch. Toxicol. 2012, 86, 1107-1115. [CrossRef] [PubMed]

35. Chen, N.; Song, Z.M.; Tang, H.; Xi, W.S.; Cao, A.; Liu, Y.; Wang, H. Toxicological Effects of Caco-2 Cells Following Short-Term and Long-Term Exposure to Ag Nanoparticles. Int. J. Mol. Sci. 2016, 17. [CrossRef] [PubMed]

36. Setyawati, M.I.; Tay, C.Y.; Leong, D.T. Mechanistic Investigation of the Biological Effects of $\mathrm{SiO}_{2}, \mathrm{TiO}_{2}$, and ZnO Nanoparticles on Intestinal Cells. Small 2015, 11, 3458-3468. [CrossRef] [PubMed]

37. Chen, H.; Zhao, R.; Wang, B.; Cai, C.; Zheng, L.; Wang, H.; Wang, M.; Ouyang, H.; Zhou, X.; Chai, Z.; et al. The effects of orally administered $\mathrm{Ag}, \mathrm{TiO}_{2}$ and $\mathrm{SiO}_{2}$ nanoparticles on gut microbiota composition and colitis induction in mice. NanoImpact 2017, 8, 80-88. [CrossRef]

38. van den Brule, S.; Ambroise, J.; Lecloux, H.; Levard, C.; Soulas, R.; De Temmerman, P.J.; Palmai-Pallag, M.; Marbaix, E.; Lison, D. Dietary silver nanoparticles can disturb the gut microbiota in mice. Part. Fibre Toxicol. 2016, 13, 38. [CrossRef] [PubMed]

39. Ohno, M.; Nishida, A. Nanoparticle curcumin ameliorates experimental colitis via modulation of gut microbiota and induction of regulatory T cells. PLoS ONE 2017, 12, e0185999. [CrossRef] [PubMed]

40. Han, X.; Geller, B.; Moniz, K.; Das, P.; Chippindale, A.K.; Walker, V.K. Monitoring the developmental impact of copper and silver nanoparticle exposure in Drosophila and their microbiomes. Sci. Total Environ. 2014, 487, 822-829. [CrossRef] [PubMed]

41. Guo, Z.; Martucci, N.J.; Liu, Y. Silicon dioxide nanoparticle exposure affects small intestine function in an in vitro model. Nanotoxicology 2018, 12, 485-508. [CrossRef] [PubMed] 
42. Guo, Z.; Martucci, N.J.; Moreno-Olivas, F.; Tako, E.; Mahler, G.J. Titanium Dioxide Nanoparticle Ingestion Alters Nutrient Absorption in an In Vitro Model of the Small Intestine. NanoImpact 2017, 5, 70-82. [CrossRef] [PubMed]

43. Richter, J.W.; Shull, G.M.; Fountain, J.H.; Guo, Z.; Musselman, L.P.; Fiumera, A.C.; Mahler, G.J. Titanium dioxide nanoparticle exposure alters metabolic homeostasis in a cell culture model of the intestinal epithelium and Drosophila melanogaster. Nanotoxicology 2018, 12, 390-406. [CrossRef] [PubMed]

44. Ong, C.; Lee, Q.Y.; Cai, Y.; Liu, X.; Ding, J.; Yung, L.Y.; Bay, B.H.; Baeg, G.H. Silver nanoparticles disrupt germline stem cell maintenance in the Drosophila testis. Sci. Rep. 2016, 6, 20632. [CrossRef] [PubMed]

45. Kalyanaraman, B.; Darley-Usmar, V.; Davies, K.J.; Dennery, P.A.; Forman, H.J.; Grisham, M.B.; Mann, G.E.; Moore, K.; Roberts, L.J., 2nd; Ischiropoulos, H. Measuring reactive oxygen and nitrogen species with fluorescent probes: Challenges and limitations. Free Radic. Biol. Med. 2012, 52, 1-6. [CrossRef] [PubMed]

46. Sykiotis, G.P.; Bohmann, D. Keap1/Nrf2 signaling regulates oxidative stress tolerance and lifespan in Drosophila. Dev. Cell 2008, 14, 76-85. [CrossRef] [PubMed]

47. Itoh, K.; Chiba, T.; Takahashi, S.; Ishii, T.; Igarashi, K.; Katoh, Y.; Oyake, T.; Hayashi, N.; Satoh, K.; Hatayama, I.; et al. An Nrf2/small Maf heterodimer mediates the induction of phase II detoxifying enzyme genes through antioxidant response elements. Biochem. Biophys. Res. Commun. 1997, 236, 313-322. [CrossRef] [PubMed]

48. Itoh, K.; Tong, K.I.; Yamamoto, M. Molecular mechanism activating Nrf2-Keap1 pathway in regulation of adaptive response to electrophiles. Free Radic. Biol. Med. 2004, 36, 1208-1213. [CrossRef] [PubMed]

49. Shi, X.; Zhang, Y.; Zheng, J.; Pan, J. Reactive oxygen species in cancer stem cells. Antioxid. Redox Signal. 2012, 16, 1215-1228. [CrossRef] [PubMed]

50. Liang, R.; Ghaffari, S. Stem cells, redox signaling, and stem cell aging. Antioxid. Redox Signal. 2014, 20, 1902-1916. [CrossRef] [PubMed]

(C) 2018 by the authors. Licensee MDPI, Basel, Switzerland. This article is an open access article distributed under the terms and conditions of the Creative Commons Attribution (CC BY) license (http:/ / creativecommons.org/licenses/by/4.0/). 